\title{
Angiopoietin-like protein 3 and 4 in obesity, type 2 diabetes mellitus, and malnutrition: the effect of weight reduction and realimentation
}

\author{
Anna Cinkajzlová 1,2, Miloš Mrázz,3, Zdeňka Lacinová1,2, Jana Kloučková1,2, Petra Kaválková2, Helena Kratochvílová1,2,

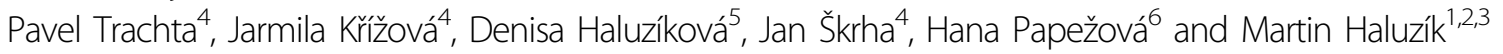

\begin{abstract}
Background Angiopoietin-like proteins (ANGPTLs) 3 and 4 are circulating factors that participate in the regulation of lipid and glucose metabolism.

Subjects and methods We measured serum ANGPTL3 and 4 levels in 23 patients with obesity, 40 patients with obesity and type 2 diabetes mellitus (T2DM), 22 patients with anorexia nervosa (AN), 15 subjects undergoing 72-h fasting, and 12 patients with short bowel syndrome (SBS), and their changes after very-low-calorie diet (VLCD), bariatric surgery, partial realimentation, acute fasting, and parenteral nutrition in order to assess their possible role in metabolic regulations.
\end{abstract}

Results Serum ANGPTL4 levels were higher in obese subjects without/with T2DM ( $94.50 \pm 9.51$ and $134.19 \pm 7.69$ vs. $50.34 \pm 4.22 \mathrm{ng} / \mathrm{ml}, p<0.001)$ and lower in subjects with AN relative to healthy control subjects (38.22 $\pm 4.48 \mathrm{vs}$. 65.80 $\pm 7.98 \mathrm{ng} / \mathrm{ml}, p=0.002$ ), while serum ANGPTL3 levels demonstrated inverse tendency. Nutritional status had no effect on ANGPTL3 and 4 mRNA expression in adipose tissue. Fasting decreased ANGPTL3 and increased ANGPTL4 levels, while VLCD reduced only ANGPTL3. Bariatric surgery and realimentation of AN or SBS patients had no effect on either ANGPTL. Multiple regression analysis identified BMI as an independent predictor of ANGPTL3; and BMI and HbA ${ }_{1 c}$ as independent predictors of ANGPTL4, respectively.

Conclusions Taken together, our data suggest that serum ANGPTL3 and 4 levels are influenced by nutritional status and fasting and could be involved in the metabolic disturbances present in obesity and AN.

\section{Introduction}

Angiopoietin-like proteins (ANGPTLs) 3 and 4 are members of the ANGPTL protein family named

\footnotetext{
Correspondence: Martin Haluzík (halm@ikem.cz)

${ }^{1}$ Centre for Experimental Medicine, Institute for Clinical and Experimental Medicine, Prague, Czech Republic

${ }^{2}$ Department of Medical Biochemistry and Laboratory Diagnostics, First Faculty of Medicine and General University Hospital, Charles University in Prague, Prague, Czech Republic
}

Full list of author information is available at the end of the article according to their structural similarity with angiopoietins $^{1}$. However, ANGPTLs do not bind to angiopoietinspecific receptors ${ }^{2}$ and are thus still considered orphan ligands. Both ANGPTL4 (peroxisome proliferator-activated- $\gamma$ angiopoietin-related protein or fasting-induced adipose factor) and ANGPTL3 have been shown to affect a number of biological processes, including angiogenesis $^{3,4}$, hematopoietic stem cell activity ${ }^{5,6}$, cancer cell

\section{(c) The Author(s) 2018}

(c) (i) Open Access This article is licensed under a Creative Commons Attribution 4.0 International License, which permits use, sharing, adaptation, distribution and reproduction cc in any medium or format, as long as you give appropriate credit to the original author(s) and the source, provide a link to the Creative Commons license, and indicate if changes were made. The images or other third party material in this article are included in the article's Creative Commons license, unless indicated otherwise in a credit line to the material. If material is not included in the article's Creative Commons license and your intended use is not permitted by statutory regulation or exceeds the permitted use, you will need to obtain permission directly from the copyright holder. To view a copy of this license, visit http://creativecommons.org/licenses/by/4.0/. 
invasion $^{7,8}$ as well as regulation of lipid and glucose metabolism ${ }^{9,10}$.

ANGPTL3 and 4 are released into systemic circulation primarily from the liver and act in a number of tissues, including white adipose tissue (WAT) ${ }^{2}$. In spite of their similar structure with $31 \%$ amino-acid sequence identity $^{11}$, their biological actions differ according to underlying conditions ${ }^{12-14}$. ANGPTL3 is upregulated by liver X receptor, which serves as a sensor of cholesterol metabolism and lipid biosynthesis ${ }^{15,16}$, while ANGPTL4 is a downstream target of peroxisome proliferator-activated receptors that modulate lipid metabolism, insulin sensitivity, and adipocyte differentiation. Both ANGPTL3 and ANGPTL4 also inhibit lipoprotein lipase (LPL) and stimulate lipolysis ${ }^{17}$.

In spite of these data, the exact roles of ANGPTL3 and 4 in glucose and lipid metabolism in humans are still only partially understood. In our study, we therefore explored the changes of ANGPTL3 and ANGPTL4 circulating levels and their mRNA expression in subcutaneous adipose tissue (SAT) of obese subjects with and without type 2 diabetes mellitus (T2DM) as well as chronically malnourished individuals with anorexia nervosa (AN) or short bowel syndrome (SBS) as models of severe malnutrition of different etiology. We further evaluated the effects of selected energy balance-modifying interventions, including acute fasting, very-low-calorie diet (VLCD), bariatric surgery, and partial enteral and parenteral realimentation on both ANGPTLs in order to evaluate their potential role in metabolic improvements associated with these procedures and gain more insight into their suggested metabolic regulatory functions.

\section{Materials and methods}

\section{Study subjects and interventions}

Twenty-three patients with simple obesity (OB group), 40 obese individuals with T2DM (T2DM group, 27 of which underwent VLCD and 13 bariatric surgery), 22 patients with AN (AN group), 15 subjects undergoing a 72-h fast to rule out organic hyperinsulinism, and 12 subjects with SBS (SBS group) were enrolled into the study. Two age-matched healthy lean control groups (1 with 22 subjects for $\mathrm{OB}$ and T2DM individuals and a second one with 15 subjects for AN group) were included as well. Written informed consent was obtained from each subject prior to enrollment. The study was approved by the Human Ethics Review Board, First Faculty of Medicine and General University Hospital, Prague, Czech Republic.

\section{Anthropometric examination, blood, and adipose tissue sampling}

All subject included in the study were measured and weighted, and their body mass index (BMI) was calculated. Blood samples for biochemical and hormonal measurements were taken after overnight fasting and were centrifuged for $10 \mathrm{~min}$ at $1000 \times g$ within $30 \mathrm{~min}$ from collection. Aliquots were stored at $-80^{\circ} \mathrm{C}$.

Samples of SAT were obtained by needle aspiration biopsy from abdominal region or from laparoscopic channel or laparotomy in case of surgery after overnight fasting. In subjects undergoing bariatric surgery visceral adipose tissue (VAT) samples were taken from abdominal cavity at the beginning of the procedure. Samples were subsequently stored at $-80^{\circ} \mathrm{C}$.

\section{Very-low-calorie diet}

Twenty-three obese patients and 27 obese patients with T2DM underwent a 3-week VLCD period. The VLCD diet consisted of $50 \mathrm{~g}$ of carbohydrates (15-20 g in monoand disaccharides, and 30-35 $\mathrm{g}$ in polysaccharides), $20 \mathrm{~g}$ of lipids (6-8 g unsaturated and 12-14 g saturated), $55 \mathrm{~g}$ of proteins, and $20 \mathrm{~g}$ of fiber accounting for a total energy content of $2500 \mathrm{~kJ} /$ day. Anthropometric measurements and blood and SAT samples were taken before and after VLCD.

\section{Bariatric surgery}

Thirteen obese patients with T2DM underwent bariatric surgery. The procedures included gastric plication (10 subjects), gastric banding (2 subjects), and gastric bypass ( 1 subject). Anthropometric measurements and blood and SAT samples were taken at baseline (visit 1) and 1 (visit 2), 6 (visit 3), and 12 months after surgery (visit 4). In addition to SAT obtained during each visit, samples of SAT and VAT were taken during surgery for the evaluation of depot-specific differences.

\section{Partial realimentation in subjects with AN}

Twenty-two patients with AN were included into this substudy. The diagnosis of eating disorder was based on Diagnostic Statistical Manual IV. The mean duration of AN was $9.0 \pm 1.8$ years. Subjects were free of any medication for more than 3 months prior to the beginning of the study. The realimentation program has been described in more detail previously ${ }^{18}$. During the realimentation period (46 \pm 2 days) the daily energy content was $9630 \mathrm{~kJ} /$ day. Anthropometric and blood samples were taken at baseline and after realimentation.

\section{Fasting in subjects with functional hypoglycemia}

Fifteen non-obese non-diabetic subjects with suspected hypoglycemic episodes underwent a supervised 72-h fasting test to rule out organic hyperinsulinism, all of them with negative results. ANGPTLs were measured at the beginning (V1) and end of fasting (i.e., after $72 \mathrm{~h}-\mathrm{V} 2$ ) and $2 \mathrm{~h}$ after realimentation (V3). 


\section{Parenteral nutrition in SBS subjects}

Twelve patients with SBS on parenteral nutrition were included into this substudy. Blood samples were taken at baseline (V1) and 14 days (V2), 6 (V3), and 12 months (V4) after hospitalization.

\section{Hormonal and biochemical assays}

Serum ANGPTL3 and ANGPTL4 levels were measured by sandwich enzyme immunoassays using commercial ELISA kits (BioVendor, Brno, Czech Republic, Brno). The whole assays, including serum dilution were done according to the manufacturer's protocol. Sensitivity was $1.08 \mathrm{ng} / \mathrm{ml}$ for ANGPTL3 and $0.173 \mathrm{ng} / \mathrm{ml}$ for ANGPTL4. Serum leptin concentrations were measured by commercial ELISA kit (Biovendor). Sensitivity was $0.2 \mathrm{ng} / \mathrm{ml}$. Serum high-sensitivity C-reactive protein (hsCRP) levels were measured by high-sensitive ELISA (Bender Medsystems, Vienna, Austria) with sensitivity of $3 \mathrm{pg} / \mathrm{ml}$. Insulin concentrations were measured by RIA kit (Cis Bio International, Gif-sur-Yvette, France). Sensitivity was 2.0 $\mu \mathrm{IU} / \mathrm{ml}$. The intra- and interassay variabilities were $<5.0$ and $10.0 \%$, respectively.

Biochemical parameters (blood glucose, glycated hemoglobin- $-\mathrm{HbA}_{1 \mathrm{c}}$, total and high-density lipoprotein (HDL) cholesterol, triglycerides, and CRP) were measured and low-density lipoprotein (LDL) cholesterol was calculated by standard laboratory methods. The homeostasis model assessment (HOMA) was calculated according to equation: (fasting serum insulin $(\mathrm{mIU} / \mathrm{l}) \times$ fasting serum glucose $(\mathrm{mmol} / \mathrm{l})) / 22.5$.

\section{Quantitative real-time PCR}

SAT and VAT samples were homogenized on MagNA Lyser Instrument (Roche Diagnostics GmbH, Mannheim, Germany). Total RNA from homogenized tissue was extracted on MagNA Pure instrument using MagNa Pure Compact RNA Isolation kit (tissue; Roche Diagnostics $\mathrm{GmbH}$ ). RNA concentration was determined from absorbance at $260 \mathrm{~nm}$ on a NanoPhotometer (Implen, Munchen, Germany). Reverse transcription was performed using random primers according the manufacturer's protocol of the High-Capacity cDNA Reverse Transcription Kits (Applied Biosystems, Foster City, CA, USA). The input amount of RNA was $750 \mu \mathrm{g} /$ reaction. Gene expression was performed on a 7500 Real-Time PCR System (Applied Biosystems). For reaction a mix of TaqMan Universal PCR Master Mix II, NO AmpErase UNG (Applied Biosystems), nuclease-free water (Fermentas Life Science, Vilnius, Lithuania), and specific TaqMan gene Expression Assays (Applied Biosystems) was used. Beta-2 microglobulin was applied as endogenous reference. The formula $2^{-\mathrm{ddCt}}$ was used to calculate relative gene expression. For determination of ANGPTL4 mRNA expression diluted samples were used, while for
ANGPTL3 mRNA expression samples remained undiluted.

\section{Statistical analysis}

Statistical analysis was performed on SigmaStat 3.0 and SigmaPlot 8.0 software (SPSS Inc., Chicago, IL, USA). Anthropometric, biochemical, and hormonal results are expressed as means \pm standard error of the mean. Oneway analysis of variance (ANOVA)/one-way repeated measures (RM) ANOVA followed by Holm-Sidak test, one-way ANOVA on ranks/one-way RM ANOVA on ranks followed by Dunn's method, unpaired $t$-test or Mann-Whitney rank sum test, and paired-test or Wilcoxon signed-rank were used for the assessment of intergroup differences, as appropriate. Spearman or Pearson correlation test was used to calculate the relationships between serum ANGPTL3 and 4 levels or mRNA expression and other parameters. Combined groups of all study subjects with age-matched control subjects were used for correlation analysis. Statistical significance was assigned to $p<0.05$. Multiple linear regression analysis using the backward stepwise variable selection method was performed using parameters with significant results from Spearman or Pearson correlation test.

\section{Results}

\section{Very-low-calorie diet}

At baseline, obese patients with and without T2DM had higher BMI, insulin, leptin, hsCRP, and HOMA index relative to control subjects, while T2DM patients also showed increased blood glucose, $\mathrm{HbA}_{1 \mathrm{c}}$, and triglycerides, and reduced HDL cholesterol (Table 1). Non-diabetic subjects had lower BMI, blood glucose, $\mathrm{HbA}_{1 \mathrm{c}}$, HOMA index, triglycerides, leptin, and hsCRP, and higher HDL cholesterol at baseline compared to diabetics enrolled in the VLCD substudy.

VLCD reduced BMI, blood glucose, HOMA index, leptin, and hsCRP in both groups with additional decrease in total, HDL, and LDL cholesterol in T2DM subjects (Table 1).

\section{Bariatric surgery}

At baseline, obese patients with T2DM enrolled in the bariatric surgery substudy had higher BMI, blood glucose, $\mathrm{HbA}_{1 \mathrm{c}}$, triglycerides, insulin, HOMA index, leptin, and hsCRP, and reduced HDL cholesterol relative to control subjects (Table 2).

Bariatric surgery significantly reduced $\mathrm{BMI}, \mathrm{HbA}_{1 \mathrm{c}}$, triglycerides, leptin, and hsCRP levels, and these changes lasted until one year after the procedure (Table 2). A temporary improvement was also observed in fasting glucose, insulin, and HOMA index. 


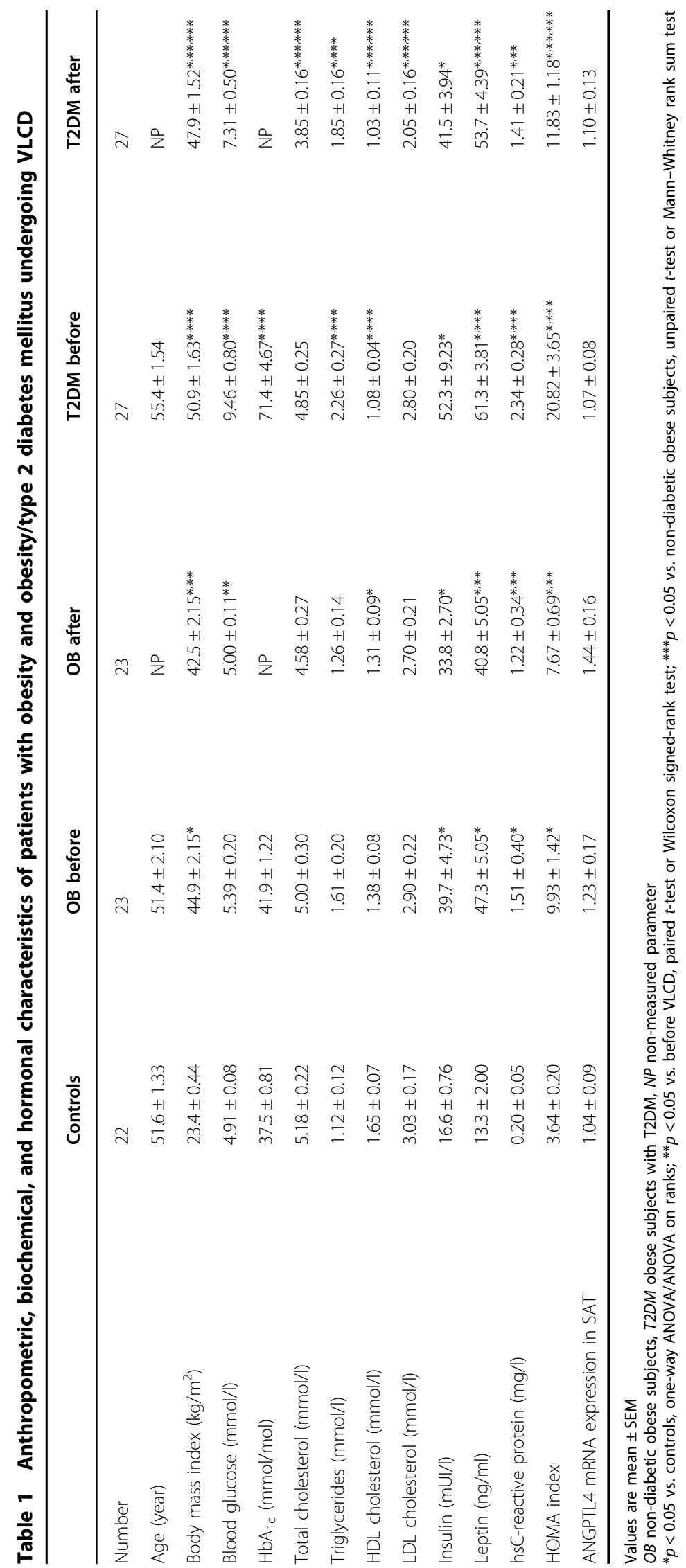




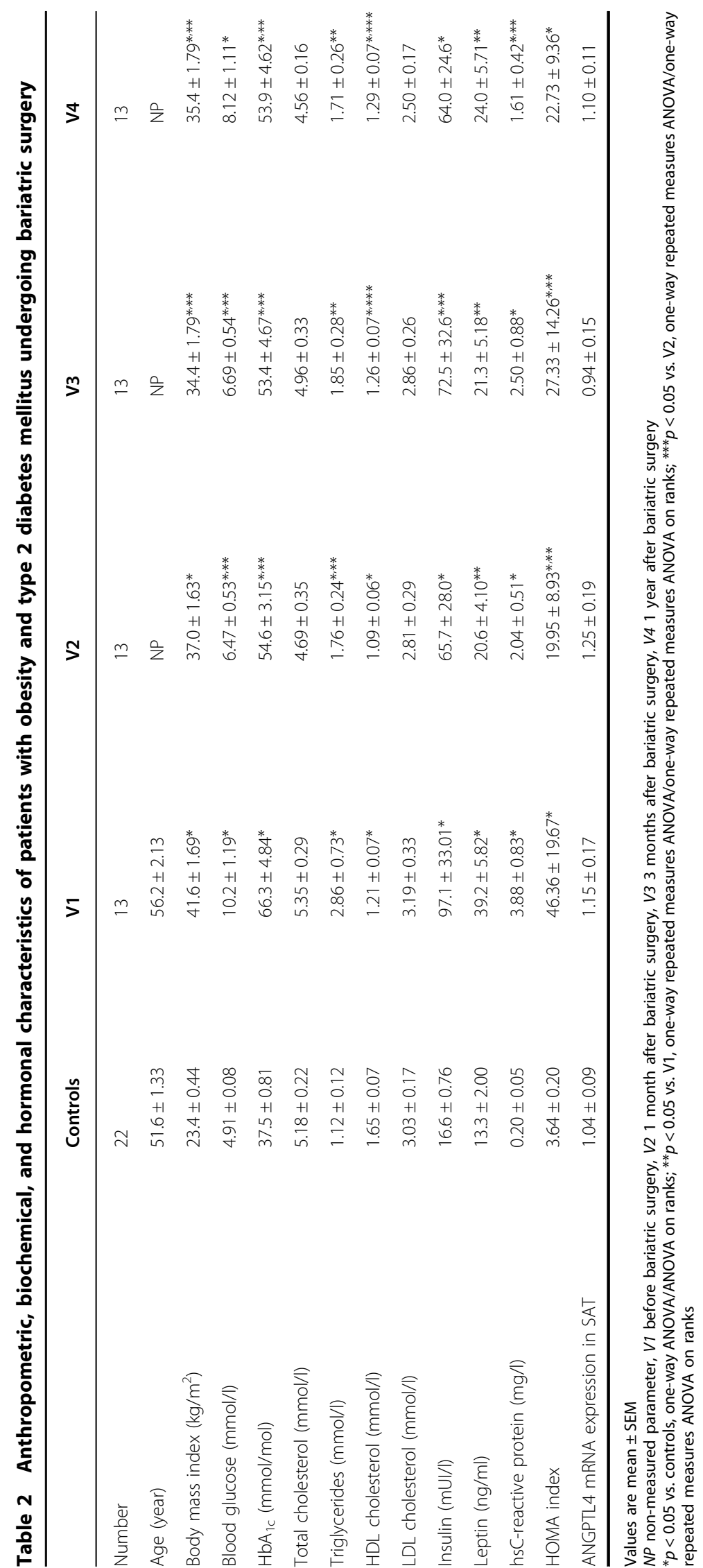



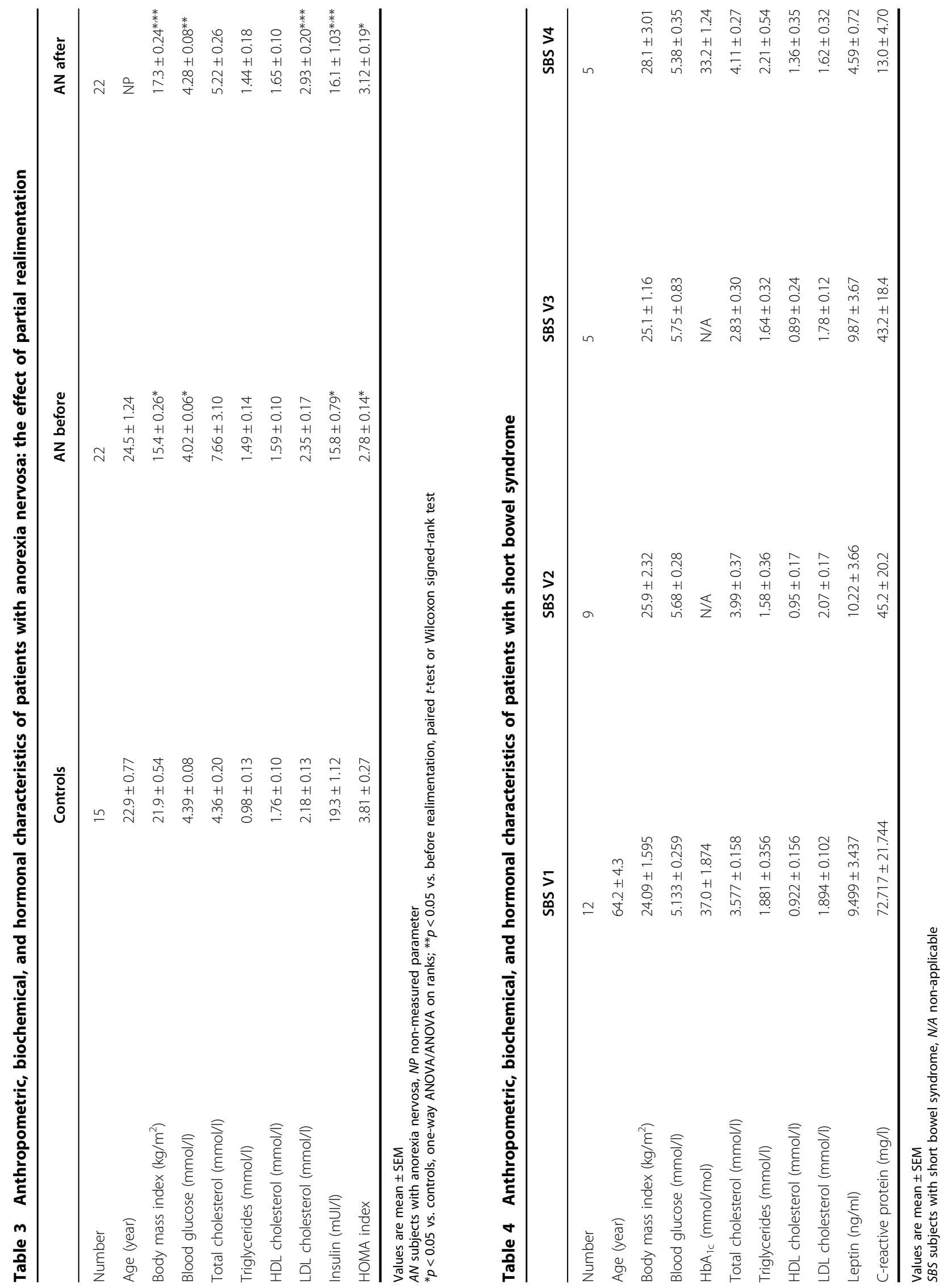


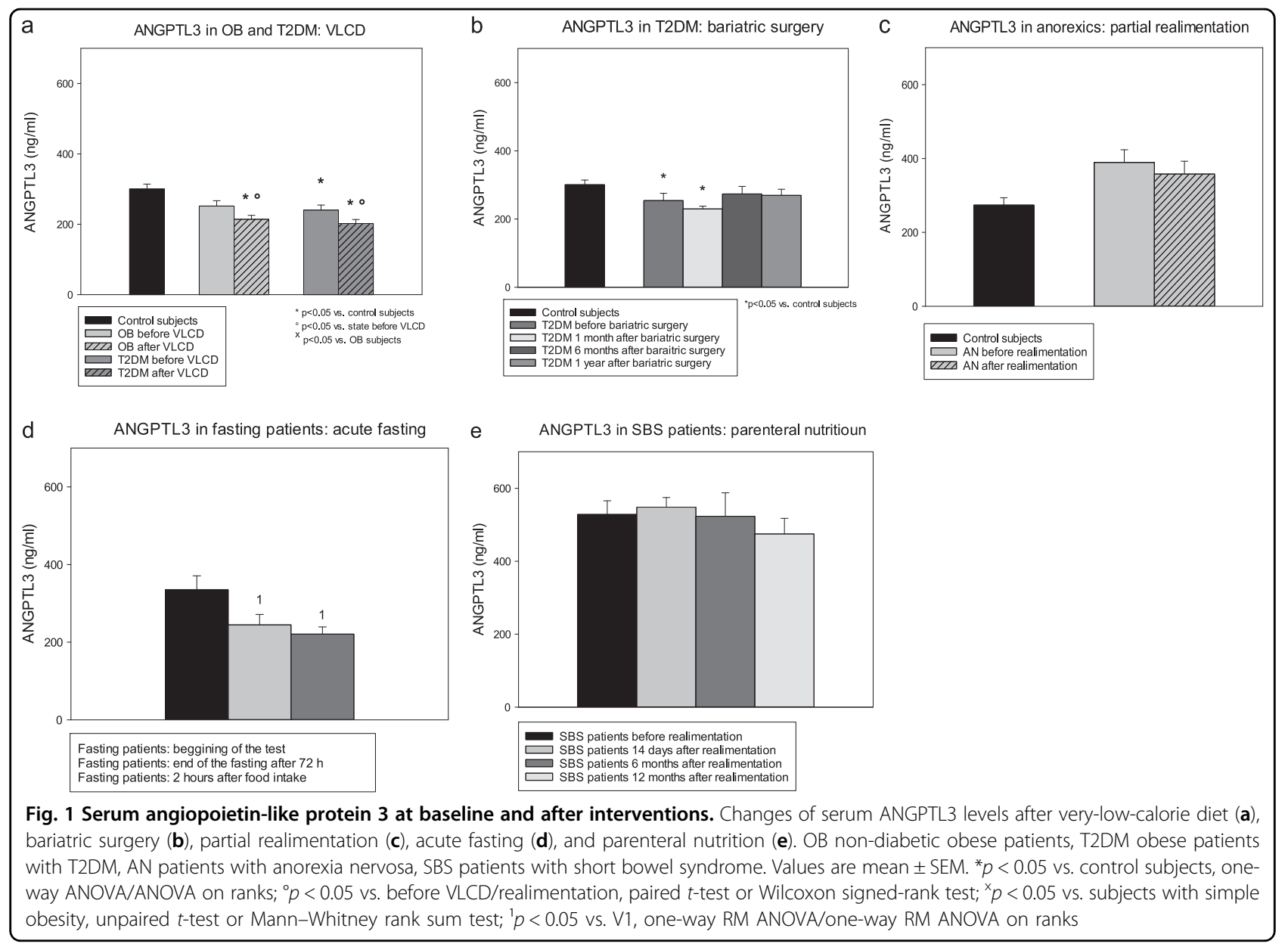

Different types of malnutrition/negative energy balanceAN, SBS, and acute fasting

At baseline, patients with AN had lower BMI, blood glucose, insulin, and HOMA index compared with control subjects with a significant increase after realimentation (Table 3). Upon enrollment, subjects with SBS had normal BMI and glucose levels. Parenteral nutrition had no significant effect on any of the measured parameters, although a trend to increased BMI and reduced CRP was observed (Table 4). Patients undergoing acute fasting had normal baseline fasting glucose and lipid levels (data not shown).

\section{Effect of interventions on serum ANGPTL3 and 4 levels and adipose tissue mRNA expression}

In the VLCD substudy, serum ANGPTL3 levels were at baseline lower in obese diabetic, but not in nondiabetic, patients compared with healthy controls (Fig. 1a), while ANGPTL4 was elevated in both obese subjects with and without T2DM (Fig. 2a). ANGPTL4 levels were also higher in T2DM relative to non-diabetic subjects. VLCD decreased ANGPTL3 levels
(Fig. 1a), while having no effect on serum ANGPTL4 (Fig. 2a).

At baseline, obese diabetics enrolled in the bariatric surgery substudy had decreased ANGPTL3 (Fig. 1b) and increased ANGPTL4 levels (Fig. 2b) relative to control subjects. Bariatric surgery did not significantly affect ANGPTL3 levels (Fig. 2a), while reducing serum ANGPTL4 at months 6 and 12 relative to month 1 after procedure (Fig. 2b).

Before intervention, patients with AN showed lower ANGPTL4 and a trend toward higher ANGPTL3 levels relative to control subjects (Figs. 1c and 2c). Partial realimentation had no effect on serum ANGPTLs. Acute fasting decreased serum ANGPTL3 and simultaneously increased ANGPTL4 concentrations with subsequent food intake having no effect during the $2 \mathrm{~h}$ post fasting (Figs. 1d and 2d). Throughout the whole year parenteral nutrition did not influence either ANGPTL in SBS subjects (Figs. 1e and 2e).

ANGPTL4 mRNA expression in SAT was affected neither by the presence of obesity/T2DM nor by weightreducing interventions (Fig. 2f, g). When compared between SAT and VAT no difference in ANGPTL4 


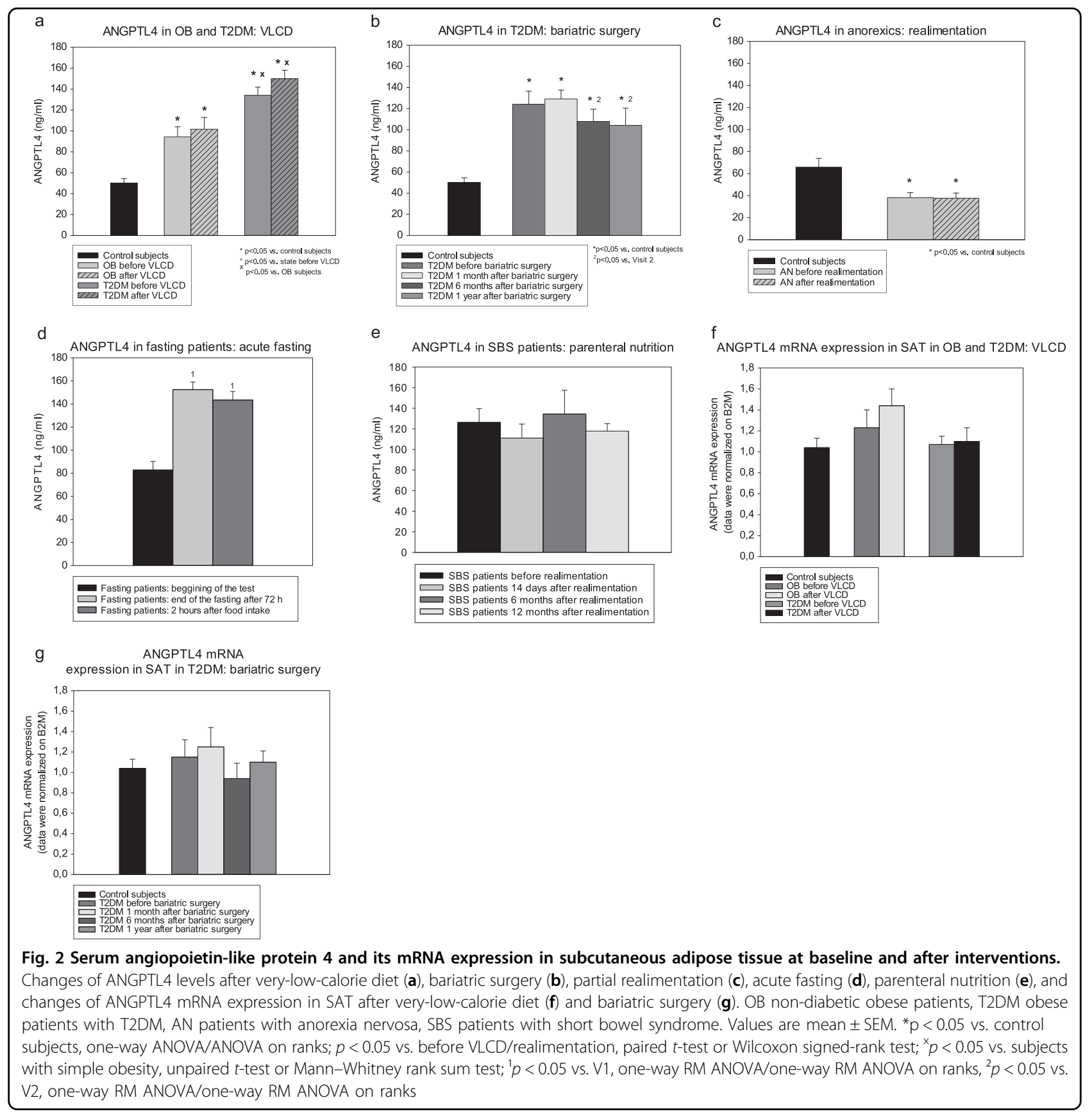

mRNA expression was found in obese patients with T2DM undergoing bariatric surgery $(p=0.092)$. In contrast, ANGPTL3 mRNA expression was almost undetectable in adipose tissue (data not shown).

\section{Relationship of ANGPTLs to other parameters}

In the combined group of all study subjects except SBS subjects, ANGPTL3 levels positively correlated with HDL cholesterol and inversely with BMI, blood glucose, triglycerides, insulin, HOMA index, and leptin (Supplemental Table 1). Multiple linear regression analysis revealed that BMI was the only independent predictor for ANGPTL3 levels (adjusted $R=0.153, p<0.001$ ).

ANGPTL4 levels positively correlated with BMI, blood glucose, $\mathrm{HbA}_{1 \mathrm{c}}$, triglycerides, insulin, leptin, hsCRP, and HOMA index, and negatively with HDL cholesterol (Supplemental Table 1). Multiple linear regression identified BMI $(p>0.001)$ and $\mathrm{HbA}_{1 \mathrm{c}}(p>0.001)$ as independent predictors of serum ANGPTL4 (adjusted $R=0.552$, $p<0.001)$.

ANGPTL3 levels correlated inversely with ANGPTL4 levels $(R=-0.436, p>0.001)$. No significant relationship 
with any of the studied parameters was found for ANGPTL4 mRNA expression.

\section{Discussion}

ANGPTL3 and ANGPTL4 have multiple functions in human body, including a possible role in the regulation of glucose and lipid homeostasis. It is therefore relevant to study changes of their circulating levels and adipose tissue mRNA expression in patients with different nutritional statuses and disturbances in glucose metabolism. In this study we show an opposite association pattern of serum ANGPTL3 and ANGPTL4 with body weight, diabetes status, and parameters of glucose control across a wide range of BMI as well as their different reactions to shortand long-term weight-modifying interventions. Both ANGPTL3 and 4 are potent regulators of triglyceride metabolism influencing triglyceride trafficking and circulating triglyceride levels by selectively inhibiting LPL in key metabolic tissues, including WAT, skeletal muscle, and heart. According to the recently proposed "3-4-8" model based primarily on experimental data ANGPTL4, a selective inhibitor of WAT LPL, is upregulated during fasting shunting thus triglycerides from WAT to skeletal and heart muscle. In contrast, the complex of ANGPTL3 and ANGPTL8 selectively inhibits skeletal and heart LPL, and while ANGPLT3 was reported not to be influenced by nutritional status, ANGPTL8 is upregulated after feeding redirecting the triglyceride flux away from heart and skeletal muscle and into $\mathrm{WAT}^{19}$. Here we partially confirm this model, as acute fasting in our subjects almost doubled baseline serum ANGPTL4 levels, which is in line with previous evidence ${ }^{11,20,21}$. Surprisingly, it also significantly decreased serum ANGPTL3, which is in contrast with former data indicating only minimal nutritional regulation of ANGPTL3 synthesis and secretion ${ }^{11}$. However, previous data were derived from in vitro and animal experiments and up to now have not been validated in human subjects. Our results are thus the first to suggest the possibility that ANGPTL3 levels are influenced by acute nutritional status in humans changing in an opposite direction to ANGPTL4. Interestingly, acute refeeding did not affect either ANGPTL levels, most probably owing to the rather short examination period $(2 \mathrm{~h}$ post refeeding), as in a recent study a significant change in ANGPTL3 and 4 levels could be observed only after 4-6 h postprandially; however, the present trend to reduced postprandial levels corresponds with the published results ${ }^{22}$.

In contrast to acute fasting, chronic malnutrition in subjects with AN had a completely opposite effect on both factors. It markedly decreased serum ANGPTL4 and tended to increase ANGPTL3 as compared with healthy controls. Analogously, the presence of obesity significantly increased ANGPTL4 while tending to reduce
ANGPTL3 suggesting body weight as one of the main regulators of ANGPTL levels. This was confirmed by correlation analysis across the whole BMI spectrum with strong positive correlation for ANGPLT4 and BMI and a weaker inverse correlation for ANGPTL3 and BMI, respectively. Interestingly, the presence of T2DM further strengthened the association for ANGPTL4 beyond the sole effect of increased BMI in T2DM compared with OB group, as evidenced by the independent positive association of its levels with $\mathrm{HbA}_{1 \mathrm{C}}$. The influence of T2DM on serum ANGPTL3 was less pronounced; however, significantly decreased ANGPTL3 concentrations in the diabetic group as compared with only an insignificant reduction trend in subjects with simple obesity might indicate a causal interconnection between T2DM and circulating ANGPTL3.

The positive association between ANGPTL4 and BMI potentiated by T2DM in our study confirms previous data $^{23,24}$ as does the much tighter relationship between ANGPTL4 and metabolic parameters compared with ANGPTL $3{ }^{12}$. However, exact mechanisms responsible for these relationships remain largely unidentified. In contrast to the original hypothesis of free fatty acids as main PPAR ligands being the primary regulators of ANGPTL4 synthesis ${ }^{25}$, recent data suggest low-grade inflammation as a more important contributor to increased ANGPTL4 levels in T2DM and the metabolic syndrome $^{23}$. Here we have shown a strong positive correlation between serum ANGPTL4 and hsCRP as a marker of low-grade inflammation that, nevertheless, could not be confirmed in multiple regression analysis. Similarly unclear is the potential role of ANGPTL4 in the development of T2DM and its complications. On one hand, increased ANGPTL4 levels are through inhibition of LPL associated with worsened lipid profile (decreased HDL and increased triglycerides), which was also confirmed in our study ${ }^{23,26}$. On the other hand, overexpression of ANGPTL4 was in experimental studies shown to decrease blood glucose and improve glucose tolerance and insulin resistance ${ }^{26,27}$. The recently proposed model of selective inhibition of LPL in WAT by ANGPTL4 with subsequent rerouting of triglycerides into heart and skeletal muscle might constitute a positive (increased energy influx into tissues) as well as negative mechanism (ectopic accumulation of lipids resulting in increased insulin resistance); to clarify this issue, more indepth mechanistic studies are required.

Unlike ANGPTL4, the relationship of ANGPTL3 and obesity and T2DM is less clear. In a recent trial in Middle Eastern population, Abu-Farha et al. ${ }^{24}$ showed increased ANGPTL3 levels only in obese non-diabetic subjects as compared to healthy normal-weight controls, whereas in the diabetic group obesity did not affect ANGPTL3 concentrations. The presence of T2DM had no effect on 
ANGPTL3 regardless of body weight. In contrast, no difference in circulating ANGPTL3 between normal and overweight individuals was observed in a study on pediatric population in $\mathrm{Korea}^{28}$. Interestingly, Zhao et al. $^{29}$ reported decreased ANGPTL3 levels in female subjects with T2DM relative to their non-diabetic counterparts (while failing to find any difference in males) as well as increased ANGPTL3 in non-diabetic females compared with males. Similarly, our data show a significant reduction in serum ANGPTL3 in both diabetic groups (VLCD and bariatric surgery) compared to healthy controls, whereas simple obesity was associated only with a nonsignificant trend to reduction. This somehow surprising finding might be partially explained by the substantial prevalence of females in the T2DM subgroup (80\%-32 females out of 40 subjects). Another factor contributing to this effect might be severe hyperinsulinemia in diabetic subjects, as high levels of insulin were shown to reduce systemic ANGPTL3 by decreasing its liver expression ${ }^{30}$. These data are of particular interest in the light of recent works showing improved insulin sensitivity after Angptl3 gene silencing and decreased atherosclerotic development in experimental animals along with improved lipid profile in humans treated with a selective ANGPTL3 inhibitor suggesting thus a possible protective effect of reduced ANGPTL3 levels in $\mathrm{T}^{2} \mathrm{DM}^{31-33}$.

To date, only limited data on the effect of longer-term weight reduction on serum ANGPTL4 are available, while being virtually non-existent for ANGPTL3. Three days of VLCD (471 kcal/day) increased circulating ANGPTL4 in healthy lean subjects as well as overweight T2DM individuals $^{25}$, while 8 weeks of VLCD $(600 \mathrm{kcal} /$ day) raised ANGPTL4 levels by $9 \%$ in otherwise healthy obese subjects $^{34}$. Our data show a similar, albeit nonsignificant, trend for ANGPTL4 after 3 weeks of VLCD $(600 \mathrm{kcal} /$ day $)$ in both obese and T2DM subjects, while serum ANGPTL3 was conversely reduced in both study groups. Fasting-stimulated elevation of non-esterified fatty acids was suggested as the primary mechanism responsible for the increase in ANGPTL4, while processes influencing ANGPTL3 still need to be elucidated ${ }^{34}$. Interestingly, bariatric surgery-induced weight loss resulted after 1 month in changes similar to VLCD in both ANGPTLs with subsequent reversal into opposite direction after 6 and 12 months suggesting the existence of different regulatory mechanisms of ANGPTL production for (semi-) acute and chronic weight reduction.

SBS as a model of extreme malnutrition was associated with substantially elevated levels of both ANGPTL3 and 4, which might at least partially be attributed to their initially increased proinflammatory status as evidenced by markedly high CRP levels. Interestingly, realimentation had no effect on either ANGPTL as their concentrations remained rather high even after the near-normalization of CRP hinting at the involvement of other regulatory factors than inflammation.

We also evaluated changes of ANGPTL4 mRNA expression in SAT. There were no differences between obese and control subjects or between baseline and postinterventional state, respectively. Moreover, there was no difference between mRNA expression in SAT and VAT samples. These findings together with a lack of significant correlation between mRNA expression in SAT and BMI support the previously published suggestion that adipose tissue has little impact on systemic ANGPTL4 concentrations ${ }^{35}$. Undoubtedly, this suggestion seems to be valid as well for ANGPTL3, which is expressed primarily in liver and kidney. In spite of previously published stu$\operatorname{dies}^{36,37}$, which detected no ANGPTL3 mRNA expression in adipose tissue we found a weak ANGPTL3 mRNA expression in both subcutaneous and VAT confirming thus the findings of Abu-Farha et al. ${ }^{24}$, even though the expression was on the detection limit of the used method and was detectable only in approximately $30 \%$ of samples.

In conclusion, we have demonstrated an inverse behavior of ANGPTL3 and 4 across different body weight ranges potentiated by the presence of T2DM, as well as during acute and prolonged weight-reducing interventions. Furthermore, we have shown for the first time the influence of acute fasting on serum ANGPTL3. Taken together, ANGPTL3 and 4 levels are affected by acute as well as chronical nutritional and metabolic status; however, their potential role in the pathogenesis of obesity and T2DM requires further investigation.

\section{Acknowledgements}

This study was supported by AZV 15-27863A. Part of the study subjects (obese subjects with/without obesity, patients with anorexia nervosa, and healthy control subjects) was also used for the examination of ANGPTL6 levels and the results were published in Cinkajzlova et al. (Endocr. Res. 2017;42:22-30).

\begin{abstract}
Author details
${ }^{1}$ Centre for Experimental Medicine, Institute for Clinical and Experimental Medicine, Prague, Czech Republic. '2Department of Medical Biochemistry and Laboratory Diagnostics, First Faculty of Medicine and General University Hospital, Charles University in Prague, Prague, Czech Republic. ${ }^{3}$ Diabetes Centre, Institute for Clinical and Experimental Medicine, Prague, Czech Republic. ${ }^{4}$ Third Department of Medicine-Department of Endocrinology and Metabolism, First Faculty of Medicine and General University Hospital, Charles University in Prague, Prague, Czech Republic. ${ }^{5}$ Department of Sports Medicine, First Faculty of Medicine and General University Hospital, Charles University in Prague, Prague, Czech Republic. ${ }^{6}$ Department of Psychiatry, First Faculty of Medicine and General University Hospital, Charles University in Prague, Prague, Czech Republic
\end{abstract}

Conflict of interest

The authors declare that they have no conflict of interest.

\section{Publisher's note}

Springer Nature remains neutral with regard to jurisdictional claims in published maps and institutional affiliations. 
Supplementary Information accompanies this paper at (https://doi.org/ 10.1038/s41387-018-0032-2).

Received: 25 December 2017 Revised: 6 February 2018 Accepted: 26 February 2018

Published online: 25 April 2018

\section{References}

1. Fagiani, E. \& Christofori, G. Angiopoietins in angiogenesis. Cancer Lett. 328, 18-26 (2013).

2. George, J. Angiopoietin-like proteins: another player in the metabolic field. J. Hepatol. 44, 832-834 (2006).

3. Oike, Y., Yasunaga, K. \& Suda, T. Angiopoietin-related/angiopoietin-like proteins regulate angiogenesis. Int. J. Hematol. 80, 21-28 (2004).

4. $\mathrm{Xu}$, L. et al. Angiopoietin-like 4: a double-edged sword in Atherosclerosis and ischemic stroke?. Exp. Neurol. 272, 61-66 (2015).

5. Zhang, C. C. et al. Angiopoietin-like proteins stimulate ex vivo expansion of hematopoietic stem cells. Nat. Med. 12, 240-245 (2006).

6. Schumacher, A. et al. Angpt/4 is upregulated under inflammatory conditions in the bone marrow of mice, expands myeloid progenitors, and accelerates reconstitution of platelets after myelosuppressive therapy. J. Hematol. Oncol. 8, 64 (2015).

7. Nakayama, T. et al. Expression of angiopoietin-like 4 in human gastric cancer: ANGPTL4 promotes venous invasion. Oncol. Rep. 24, 599-606 (2010).

8. Sun, Y., Long, J. \& Zhou, Y. Angiopoietin-like 4 promotes melanoma cell invasion and survival through aldolase A. Oncol. Lett. 8, 211-217 (2014).

9. Mattijssen, F. \& Kersten, S. Regulation of triglyceride metabolism by Angiopoietin-like proteins. Biochim. Biophys. Acta 1821, 782-789 (2012).

10. Sukonina, V., Lookene, A., Olivecrona, T. \& Olivecrona, G. Angiopoietin-like protein 4 converts lipoprotein lipase to inactive monomers and modulates lipase activity in adipose tissue. Proc. Natl Acad. Sci. USA 103, 17450-17455 (2006).

11. $\mathrm{Ge}, \mathrm{H}$. et al. Differential regulation and properties of angiopoietin-like proteins 3 and 4. J. Lipid Res. 46, 1484-1490 (2005).

12. Mehta, N. et al. Differential association of plasma angiopoietin-like proteins 3 and 4 with lipid and metabolic traits. Arterioscler. Thromb. Vasc. Biol. 34, 1057-1063 (2014).

13. Koster, A. et al. Transgenic angiopoietin-like (angptl)4 overexpression and targeted disruption of angpt/4 and angpt|3: regulation of triglyceride metabolism. Endocrinology 146, 4943-4950 (2005).

14. Shan, L. et al. The angiopoietin-like proteins ANGPTL3 and ANGPTL4 inhibit lipoprotein lipase activity through distinct mechanisms. J. Biol. Chem. 284, 1419-1424 (2009).

15. Kaplan, R. et al. Regulation of the angiopoietin-like protein 3 gene by LXR. J. Lipid Res. 44, 136-143 (2003).

16. Steffensen, K. R. \& Gustafsson, J. A. Putative metabolic effects of the liver $X$ receptor (LXR). Diabetes 53, S36-S42 (2004). Suppl 1.

17. Dijk, W. \& Kersten, S. Regulation of lipoprotein lipase by Angpt14. Trends Endocrinol. Metab. 25, 146-155 (2014).

18. Kavalkova, P. et al. Preadipocyte factor-1 concentrations in patients with anorexia nervosa: the influence of partial realimentation. Physiol. Res. 61, 153-159 (2012).
19. Zhang, R. The ANGPTL3-4-8 model, a molecular mechanism for triglyceride trafficking. Open Biol. 6, 150272 (2016).

20. Kersten, $\mathbf{S}$. et al. Caloric restriction and exercise increase plasma ANGPTL4 levels in humans via elevated free fatty acids. Arterioscler. Thromb. Vasc. Biol. 29, 969-974 (2009).

21. Dutton, S. \& Trayhurn, P. Regulation of angiopoietin-like protein $4 /$ fastinginduced adipose factor (Angpt/4/FIAF) expression in mouse white adipose tissue and 3T3-L1 adipocytes. Br. J. Nutr. 100, 18-26 (2008).

22. Minicocci, I. et al. Effects of angiopoietin-like protein 3 deficiency on postprandial lipid and lipoprotein metabolism. J. Lipid Res. 57, 1097-1107 (2016).

23. Tjeerdema, $\mathrm{N}$. et al. Inflammation increases plasma angiopoietin-like protein 4 in patients with the metabolic syndrome and type 2 diabetes. BMJ Open Diabetes Res. Care 2, e000034 (2014).

24. Abu-Farha, M. et al. Increased ANGPTL3, 4 and ANGPTL8/betatrophin expression levels in obesity and T2D. Lipids Health Dis. 15, 181 (2016).

25. Jonker, J. T. et al. Dietary modulation of plasma angiopoietin-like protein 4 concentrations in healthy volunteers and in patients with type 2 diabetes. Am. J. Clin. Nutr. 97, 255-260 (2013).

26. $\mathrm{Xu}, \mathrm{A}$. et al. Angiopoietin-like protein 4 decreases blood glucose and improves glucose tolerance but induces hyperlipidemia and hepatic steatosis in mice. Proc. Natl Acad. Sci. USA 102, 6086-6091 (2005).

27. Wang, Y. et al. Angiopoietin-like protein 4 improves glucose tolerance and insulin resistance but induces liver steatosis in high-fat-diet mice. Mol. Med. Rep. 14, 3293-3300 (2016).

28. Chung, H. S. et al. Circulating angiopoietin-like protein 8 (ANGPTL8) and ANGPTL3 concentrations in relation to anthropometric and metabolic profiles in Korean children: a prospective cohort study. Cardiovasc. Diabetol. 15, 1 (2016).

29. Zhao, D. et al. Different relationship between ANGPTL3 and HDL components in female non-diabetic subjects and type-2 diabetic patients. Cardiovasc Diabetol. 15, 132 (2016)

30. Nidhina Haridas, P. A. et al. Regulation of angiopoietin-like proteins (ANGPTLs) 3 and 8 by insulin. J. Clin. Endocrinol. Metab. 100, E1299-E1307 (2015).

31. Tikka, A. et al. Silencing of ANGPTL 3 (angiopoietin-like protein 3 ) in human hepatocytes results in decreased expression of gluconeogenic genes and reduced triacylglycerol-rich VLDL secretion upon insulin stimulation. Biosci. Rep. 34, e00160 (2014).

32. Gusarova, V. et al. ANGPTL3 blockade with a human monoclonal antibody reduces plasma lipids in dyslipidemic mice and monkeys. J. Lipid Res. 56, 1308-1317 (2015).

33. Dewey, F. E. et al. Genetic and pharmacologic inactivation of ANGPTL3 and cardiovascular disease. N. Engl. J. Med. 377, 211-221 (2017)

34. Cullberg, K. B. et al. Effect of weight loss and exercise on angiogenic factors in the circulation and in adipose tissue in obese subjects. Obesity (Silver Spring) 21, 454-460 (2013).

35. Mandard, S. et al. The direct peroxisome proliferator-activated receptor target fasting-induced adipose factor (FIAF/PGAR/ANGPTL4) is present in blood plasma as a truncated protein that is increased by fenofibrate treatment. J. Biol. Chem. 279, 34411-34420 (2004).

36. Romeo, S. et al. Rare loss-of-function mutations in ANGPTL family members contribute to plasma triglyceride levels in humans. J. Clin. Invest. 119, 70-79 (2009).

37. Nidhina Haridas, P. A. et al. Regulation of angiopoietin-like proteins (ANGPTLs) 3 and 8 by insulin. J. Clin. Endocrinol. Metab. 100, E1299-E1307 (2015). 